\title{
Autant en emporte la culture du coton transgénique aux États-Unis...*
}

\author{
Une perception des échanges et des présentations \\ lors des "Beltwide Cotton Conferences ", \\ Nouvelle-Orléans, Lousiane (États-Unis) \\ janvier 2010
}

Michel Fok

\section{Cirad}

UR SCA

Avenue Agropolis

TA B102/02

3498 Montpellier

France

<michel.fok@cirad.fr>

\begin{abstract}
Résumé
Les conférences 2010 du Beltwide cotton ont apporté une vision nouvelle sur les conséquences de 15 années d'utilisation massive des variétés de coton transgénique aux États-Unis. L'observation des changements dans les complexes d'ennemis des cultures de cotonnier et les solutions recherchées pour y faire face montrent que les effets positifs proclamés de l'utilisation de ces variétés se sont évanouies en termes d'efficacité du contrôle des ennemis des cultures, de réduction du coût et d'antagonisme entre voie chimique et voie biotechnologique de ce contrôle. La durabilité technique et économique des variétés transgéniques dépend de l'approche systémique et coordonnée de leur utilisation.
\end{abstract}

Mots clés : biotechnologie végétale ; coton ; États-Unis ; non-travail du sol ; plante transgénique ; résistance aux herbicides ; résistance aux organismes nuisibles.

Thèmes : amélioration génétique ; pathologie ; productions végétales.

\section{Abstract \\ Gone with the wind of transgenic cotton use in the United States A vision of the discussions and presentations at the New Orleans (Louisiana, USA) “Beltwide Cotton Conferences" in January 2010}

The Cotton Beltwide Conferences 2010 have provided a new vision of the consequences of about 15 years of widespread and non-coordinated use of transgenic cotton in the United States. The observation of the shift in pest complexes on one hand, and, on the other, the adaptation solutions being explored today show that the positive effects initially claimed look like vanished illusions in the areas of pest control effectiveness, cost reduction, and antagonism between chemical and biotech means. The technical and economic sustainability of transgenic varieties lies in a systemic and coordinated approach of their use.

Key words: cotton; pest resistance; plant biotechnology; resistance to herbicides; transgenic plants; USA; zero tillage.

Subjects: genetic improvement; pathology; vegetal productions.

es Beltwide Cotton Conferences sont organisées annuellement depuis 1983 aux États-Unis par la National
Cotton Council (NCC) dans la première semaine de janvier. Elles réunissent des chercheurs et des professionnels indus- 
triels et agricoles de la filière cotonnière du pays, tout en étant ouvertes aux participants de pays étrangers. En 2010, ces conférences ont eu lieu à la NouvelleOrléans. Le thème du coton transgénique $\mathrm{y}$ a pris une place particulière, presque quinze ans après la commercialisation des premières variétés transgéniques de grandes cultures (mais, soja, coton) intégrant un ou plusieurs gènes de Bacillus thuringiensis pour la résistance à certains ravageurs (variétés $\mathrm{Bt}$ ), ou un gène de tolérance à une matière active herbicide (surtout le glyphosate), ou bien les deux types de gènes.

Pour des conférences relatives au coton et se déroulant dans le sud des États-Unis, il est tentant de paraphraser le titre du fameux roman de Margaret Mitchell, "Gone with the wind", pour souligner l'évanouissement de quelques illusions. En effet, alors que les variétés transgéniques de coton couvrent aujourd'hui $88 \%$ des surfaces cotonnières américaines (tableau 1), les agriculteurs constatent l'émergence de la nuisibilité de ravageurs qui ne nécessitaient pas de contrôle ("nouveaux" ravageurs) et l'apparition d'un nombre croissant de plantes adventices devenues résistantes au glyphosate. Or, bien que les conférences du Beltwide restituent régulièrement depuis 1999 des travaux sur les "nouveaux" ravageurs, les changements des complexes de ravageurs auxquels font échos ces travaux ont été occultés dans une synthèse assez récente publiée par le ministère de l'Agriculture des États-Unis (FernandezCornejo et Caswell, 2006). Mais, en 2010, les conférences du Beltwide ont marqué pour la première fois une réelle attention portée aux changements observés et révélé une inquiétude des producteurs de coton aux États-Unis.

Cet article a pour but de commenter le contenu des conférences du Beltwide
2010. Nous présentons en première partie le déroulement et les thèmes abordés, puis nous restituons en deuxième partie la situation actuelle de changement des complexes d'ennemis de la culture, qui a été particulièrement discutée. La troisième partie indique les travaux de recherche engagés pour y faire face et la dernière partie souligne les illusions balayées par la situation actuelle.

\section{Des conférences}

\section{interprofessionnelles ciblées sur l'actualité cotonnière}

\section{Rencontres de chercheurs, de politiques et de professionnels de la filière}

L'institution organisatrice, la National Cotton Council (NCC), a été créée en 1938 pour promouvoir la coordination entre les acteurs privés de la production cotonnière aux États-Unis. Elle regroupe les acteurs principaux, à savoir les producteurs, les consultants (agronomes conseillers), les égreneurs, les stockeurs, les négociants et les transformateurs des graines et de la fibre. Parmi ses prérogatives, la NCC affiche aujourd'hui clairement son rôle de lobby auprès des hommes politiques. Elle exhorte régulièrement les producteurs à faire pression sur leurs élus locaux pour infléchir la politique cotonnière des États-Unis. Par le biais du site Internet de la NCC, chaque producteur peut trouver les coordonnées des élus locaux auprès de qui il peut intervenir. La NCC rend compte des faits et gestes

\section{Tableau 1. Parts des superficies (\%) en variétés transgéniques aux États-Unis en 2009.}

Table 1. Percentages of surface covered by transgenic varieties in the United States in 2009.

\begin{tabular}{lcccc}
\hline Culture & Gènes Bt seuls & Gènes HT seuls & Gènes empilés Bt + HT & Total \\
\hline Maïs & 0 & 91 & 0 & 91 \\
Coton & 17 & 23 & 48 & 88 \\
Soja & 17 & 22 & 46 & 85 \\
\hline
\end{tabular}

$\mathrm{HT}$ : tolérance à I'herbicide; $\mathrm{Bt}$ : gène $B$. thurigiensis de résistance à des ravageurs.

Source: United States Department of Agriculture-Economic Research Service (USDA-ERS) (http://www.ers.usda.gov/data/biotechCrops/). des élus sur la question du coton, de manière à maintenir la pression sur les politiques. Sa récente lettre ouverte, pour féliciter la délégation américaine aux dernières négociations de l'Organisation mondiale du commerce (OMC) à Genève, en décembre 2009, est à comprendre dans cette démarche.

Les trois jours que durent les conférences sont partagés entre la restitution des résultats de recherche (tableau 2) et les questions de production et de politique agricole. La NCC y rend également compte de ses activités, tout comme l'organisation interprofessionnelle Cotton Incorporated, important financeur de la recherche publique sur le coton. Les organisateurs sollicitent les chercheurs pour qu'ils présentent très rapidement les résultats de leurs travaux, surtout quand ils sont financés par la Cotton Incorporated. Les conférences du Beltwide permettent ainsi de connaître les derniers résultats des productions, des techniques et des recherches aux États-Unis, parfois bien avant leur publication dans les revues. Les résumés et les textes des communications ne sont pas disponibles au moment des conférences, mais les actes sont publiés sur Internet sous la forme d'enregistrements audio et vidéo, dès le mois de février, et de fichiers textes, au mois de juin. L'accès aux actes, disponibles depuis 1983, est offert à tout participant aux conférences ${ }^{1}$.

\section{Le secteur cotonnier américain en prise avec les défis intérieurs et extérieurs}

Pour aborder la question politique, les organisateurs font s'exprimer le ministre en charge de l'agriculture dans l'État où les conférences sont organisées, mais aussi des personnalités d'instances fédérales. Cette année, le ministre de la Louisiane a fustigé le projet de loi Cap \& Trade visant à réduire les émissions de gaz à effet de serre par les agriculteurs. Le commissaire chargé de la surveillance des bourses de produits agricoles a commenté le rapport de l'étude qu'il avait commanditée après la flambée des cours du coton, au début du mois de mars 2008, et qui n'a pas permis de conclure à des actes de manipulation du marché.

\footnotetext{
${ }^{1}$ http://www.cotton.org/beltwide/index.cfm? page $=$ proceedings
} 


\section{Tableau 2. Thèmes des présentations de résultats de recherche aux conférences du Beltwide 2010 (Nouvelle-Orléans).}

Table 2. Research results presentation themes at the Cotton Beltwide 2010 Conferences (New Orleans).

\begin{tabular}{|c|c|c|c|c|c|c|c|c|}
\hline Présentations & Économie & Agronomie* & Maladies & Ravageurs & Adventices & $\begin{array}{l}\text { Amélioration } \\
\text { variétale }\end{array}$ & $\begin{array}{l}\text { Technologies } \\
\text { transformation } * *\end{array}$ & Total \\
\hline Communications & 26 & 93 & 23 & 59 & 22 & 43 & 72 & 338 \\
\hline Posters & 11 & 46 & 17 & 39 & 19 & 20 & 4 & 156 \\
\hline Total & 37 & 139 & 40 & 98 & 41 & 63 & 76 & 494 \\
\hline
\end{tabular}

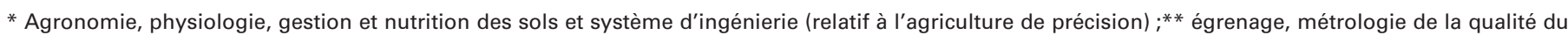
coton fibre, utilisation textile.

Les travaux coordonnés par la Cotton Incorporated indiquent que la préoccupation de la durabilité de la production cotonnière est fortement prise en compte depuis quelques années aux États-Unis. Les travaux sur le bilan énergie et carbone sont déjà assez avancés et les résultats sont exploités pour souligner que les modes de production du coton sont compatibles avec les préoccupations environnementales : la Cotton Incorporated a ainsi mis en ligne un vade-mecum pour aider à argumenter dans ce sens ${ }^{2}$. Pour autant, le manque d'eau constitue aujourd'hui l'une des plus graves menaces de la production cotonnière, notamment au Texas qui est le principal État " cotonnier ", fournissant plus de $50 \%$ de la production américaine de coton. Le défi est lancé à la fois d'une irrigation plus économe et efficiente et d'une agriculture pluviale plus productive. Cela induira des investissements supplémentaires, auxquels certains producteurs de coton ne pourront pas faire face. De ce fait, le nombre de producteurs se réduira encore alors qu'il n'est plus que de 18605 en 2007 (au lieu de 24805 en 2002 et 33640 en 1997). Les activités connexes à la production cotonnière vont donc diminuer, avec pour conséquence une perte d'emplois - estimée à 8000 emplois dans le seul État du Texas. L'exode rural qui s'ensuivra sera préjudiciable à la survie des communautés rurales actuelles.

L'autre menace concerne le changement de politique de soutien, notamment sous le coup des attaques de l'OMC. À ce propos, les travaux présentés montrent un changement notable d'attitude dans la défense des subventions américaines. Les travaux antérieurs, visant à évaluer l'effet de la politique américaine sur le

\footnotetext{
${ }^{2}$ http://www.cottoncampus.org/Cotton-Envir onmentally-Friendly-Sustainability/
}

prix mondial, concluaient à un effet dépressif de seulement 3-5\%, alors que certaines études étrangères évaluaient cet effet négatif à 15-20\%. Les nouveaux travaux présentés ont porté sur l'effet des politiques de soutien des pays concurrents sur le marché mondial. Leurs résultats sont assez étonnants : ils indiquent que la politique de prix minimum garanti en Inde, de même que la politique de contrôle des importations du coton par la Chine, ont chacune le même effet sur le prix mondial que l'ensemble de toutes les mesures de soutien appliquées aux États-Unis. Ces résultats tendraient à indiquer que ce dernier pays n'est pas plus responsable de la baisse du prix mondial que d'autres pays.

\section{Inquiétude évidente face à l'évolution des complexes parasitaires}

Au regard des sessions passées, les conférences de 2010 se singularisent par l'organisation de séances sur les changements observés dans les complexes des ennemis de la culture cotonnière. Un atelier spécifique a ainsi été animé par des chercheurs pour discuter des plantes adventices résistantes au glyphosate, utilisé massivement dans la pratique du zéro labour. L'atelier a été relayé par un panel de discussions sur le bilan des apports des variétés transgéniques, compte tenu des changements observés. Le panel était composé de deux chercheurs, l'un spécialiste de la lutte contre les ravageurs et l'autre de la lutte contre les adventices, d'un producteur du Texas et d'un consultant.

Ces deux événements ont été les plus suivis par les participants, indiquant que les producteurs, les consultants et les chercheurs ont pris pleinement conscience des changements opérés sur le terrain dans les complexes de ravageurs et d'adventices. Les citations qui suivent témoignent de leurs doutes relatifs à la poursuite des variétés de coton transgéniques :

"On est content que le coton transgénique existe, mais ça ne suffit pas pour que je dorme sur mes deux oreilles"

Consultant

"Les biotechnologies, une épée à double tranchant"

Titre de l'intervention d'un consultant "La technique du zéro labour réduit l'érosion éolienne et améliore la rétention en eau des sols, c'est très adapté au Texas, on ne voudrait pas devoir l'abandonner du fait des adventices résistants au glyphosate"

Producteur du Texas

"Ce dont on a besoin, ce sont de nouveaux produits chimiques"

Conclusion d'un malherbologue

"J'entends dire qu'il faut revenir à la culture du coton conventionnel, mais dans quelle proportion et comment s'assurer qu'une nouvelle infestation de grande ampleur de chenilles des capsules ne vienne détruire la culture?"

Expert en contrôle des ravageurs.

\section{Résultats des études} sur les complexes parasitaires

\section{Évolution des complexes de ravageurs}

Aux États-Unis, avant l'adoption du coton $\mathrm{Bt}$, les principaux ravageurs qui 
détruisaient les cultures de coton étaient les chenilles lépidoptères des capsules (Helicoverpas zea, Heliothis virescens et Pectinophora gossypiella) et le charançon des capsules Anthonomus grandis. Les ravageurs ciblés par les premières variétés de coton Bt étaient les chenilles des capsules. Leur commercialisation intervenant au moment où le programme national d'éradication du charançon lancé à la fin des années 1970 arrivait aux derniers États cotonniers, le problème des principaux ravageurs paraissait alors résolu.

L'efficacité du coton Bt contre ses ravageurs cibles est certes indéniable. L'emploi massif du coton Bt a progressivement entraîné la chute de leurs populations, à tel point que leur contrôle chimique nécessite en moyenne seulement 0,5 traitement par an à l'échelle nationale. Depuis 2003, l'utilisation du coton Bt n'induit d'ailleurs pratiquement plus de réductions du nombre de traitements contre ces ravageurs par rapport au coton conventionnel. De ce point de vue, il est étonnant de ne pas trouver d'analyse sur l'intérêt technique et économique à diminuer substantiellement le taux d'utilisation du coton Bt. Mais cette efficacité du coton Bt n'est plus suffisante et la situation actuelle est devenue compliquée. Aujourd'hui, il faut déchanter devant un changement des complexes de ravageurs, probablement dû à la très grande sélectivité des toxines Bt contre les ravageurs cibles, sélectivité par ailleurs présentée auparavant comme un avantage. Au lancement du coton Bt, les craintes concernaient en effet la résistance des ravageurs cibles aux toxines Bt et les dégâts sur la faune non ciblée (Hardee et al., 2001), mais personne ne pensait au changement de statut de nuisibilité des ravageurs non ciblés.

Trois observations soulignent ce changement des complexes de ravageurs. Premièrement, la chenille des capsules Pectinophora gossypiella est imparfaitement contrôlée. Deuxièmement, il apparaît de graves infestations de chenilles phylophages du genre Spodoptera (S. exigua et $S$. frugiperda), comme cela est aussi observé dans d'autres pays utilisateurs du coton Bt (Chine, Burkina Faso avec les espèces $S$. littoralis et S. litura). Or, le traitement chimique contre ces chenilles phylophages était inutile avant le coton Bt et il est devenu indispensable aujourd'hui. Enfin, les insectes piqueurs-suceurs sont devenus des ravageurs prépondérants, alors qu'ils ne l'étaient pas systématiquement aupara- vant. Il s'agit de deux punaises, Lygus lineolaris et Lygus besperus, d'acariens (Tetranychus spp.), d'aleurodes (Bemisia $s p p$.) et de pucerons (Aphis spp.). Même si la composition des complexes de ravageurs ayant franchi le seuil de dégâts économiques est variable selon les Etats cotonniers, les plus fortes craintes sont exprimées à l'encontre des punaises et des pucerons qui sont présents sur de nombreuses autres espèces végétales. Pour la punaise L. lineolaris, 300 espèces hôtes ont ainsi été recensées. Pour les pucerons, l'augmentation du degré d'infestation est observée depuis 2006 et a atteint un niveau en 2009 jamais observé auparavant.

Ces nouvelles émergences de ravageurs ont pour conséquence le recours accru aux insecticides chimiques. Les semenciers ont augmenté le traitement des semences ; une dizaine de produits pesticides sont ainsi utilisés, et un chercheur s'est même étonné, avec ironie, que les semences puissent encore germer dans ces conditions. En 2009, le contrôle au champ des "nouveaux " ravageurs a nécessité 6,5 traitements chimiques en moyenne dans l'ensemble des États cotonniers américains, alors que ces traitements étaient peu pratiqués. Les pertes de rendement ont également été estimées en absence de traitements chimiques ou en situation d'efficacité insuffisante de ces traitements. Les orateurs se sont même accordés à reconnaître une perte d'efficacité des produits chimiques utilisés, corrélée à l'augmentation récente de la pression des nouveaux ravageurs. Cette perte d'efficacité concerne des produits de traitement des semences et des produits de traitement foliaire de types organophosphates, carbamates et neonicotinoïdes.

Finalement, le coût du contrôle des ravageurs du cotonnier tend à augmenter depuis l'utilisation des variétés transgéniques. La sophistication du traitement des semences est l'un des facteurs de l'augmentation du coût des semences de coton Bt, qui est passé de 20 dollars US par acre en 2005 à plus de 85 dollars US par acre en 2009. À cela, il faut ajouter le coût des multiples insecticides nécessaires pour contrôler les ravageurs non ciblés du coton Bt.

L'évolution des coûts des pesticides est aussi à mettre en rapport avec celle de la structure du marché. D'une part, le nombre de firmes de phytopharmacie d'origine européenne ou américaine a drastiquement diminué : un orateur a fait remarquer qu'en 1962, on recensait
42 firmes, puis 33 en 1980, et finalement 7 en 2009. D'autre part, de nombreux produits nouveaux ont été mis sur le marché. Ces produits ont des modes d'action novateurs, mais chaque mode d'action est représenté par peu de produits commerciaux. La concurrence entre les produits est alors seulement virtuelle, car ils ne sont pas réellement substituables.

Face à l'ensemble de cette situation, la pertinence d'un retour à la culture du coton conventionnel, à un degré non explicité, est mentionnée. Il s'agit déjà d'une réalité : en 2009, il y en aurait eu 400000 acres (sur une surface totale en coton de 8,9 millions d'acres) et une superficie de 1,5 million d'acres est attendue pour 2010. La question est encore éludée par les chercheurs spécialistes du contrôle des ravageurs, ces chercheurs utilisant l'argument d'un retour possible à une forte infestation des ravageurs cibles.

\section{Évolution des complexes de plantes adventices}

Les variétés de coton transgéniques tolérantes à la matière active herbicide glyphosate couvrent aujourd'hui $71 \%$ des superficies totales de coton des États-Unis et cette part continue d'augmenter. Pourtant, depuis 2003, le phénomène de résistance des plantes adventices au glyphosate s'est progressivement étendu à tous les États producteurs de coton pour toutes les grandes cultures (coton, maïs, soja...). Ce phénomène reflète une dérive de la flore adventice, directement liée à la destruction des mauvaises herbes en zéro labour par l'utilisation d'herbicides, notamment le Roundup de Monsanto à base de glyphosate.

Les espèces adventices résistantes au glyphosate les plus fréquemment citées sont en premier lieu Conyza canadensis (horseweed) et Amaranthus palmeri ("pigweed"), qui inquiètent le plus les producteurs américains, puis Lolium rigidum ("ryegrass"), Sorghum halepense ("jobnsongrass "), Ambrosia artemisiifolia ("ragweed"). Les parcelles peuvent être totalement envahies par plusieurs d'entre elles. Une enquête réalisée en 2009 indique que le nombre d'espèces résistantes a varié de 2 à 18 selon les États cotonniers et qu'en certains comtés de ces États, $75 \%$ des champs ont été touchés et que $45 \%$ des producteurs ont recouru à l'arrachage manuel. Quel paradoxe au pays de la motorisation et où l'on clame, avec l'expansion de la pratique de l'agriculture 
de précision, que la nouvelle révolution consiste à embarquer de plus en plus d'électronique sur les machines agricoles !

Un autre phénomène inattendu a vu le jour. La culture des variétés transgéniques de soja, coton et maïs tolérantes au glyphosate a contribué à transformer ces plantes cultivées elles-mêmes en adventices. Par exemple, dans les champs de coton, les plants de soja ou de maïs issus de graines laissées après la récolte constituent les adventices les plus difficiles à maîtriser, puisque non éliminées par le glyphosate auquel elles sont tolérantes. Le coton transgénique tolérant au glyphosate est aussi une mauvaise herbe pour le soja et le maïs.

\section{Évolution des complexes parasitaires en relation avec le zéro labour}

Les variétés transgéniques tolérantes au glyphosate ont permis d'étendre la pratique du zéro labour. En zéro labour, la culture principale est semée sans travail du sol dans un couvert végétal qui a été préalablement contrôlé par herbicide. Au moment des semis, ce couvert végétal permanent induit une température du sol plus faible qu'en culture sur sol nu et une humidité plus élevée. De ce fait, les maladies fongiques des plantules se développent (fonte des semis), ainsi que les maladies fongiques foliaires après le stade plantule. Les consultants préconisent donc l'utilisation accrue de fongicides. Malgré cela, chercheurs et consultants admettent qu'il reste encore beaucoup à faire pour optimiser les techniques de traitement fongicide.

L'interaction entre l'utilisation des variétés transgéniques et les techniques culturales du zéro labour a des effets au-delà du complexe des maladies fongiques. La pression des ravageurs est aussi influencée par le couvert végétal. L'augmentation de la pression des ravageurs de début de saison, notamment les thrips, les pucerons, les acariens et les punaises, est fréquemment signalée, au point que les consultants recommandent déjà la destruction de cette végétation quelques semaines avant le semis, y compris aux alentours des parcelles à semer, en attendant que les chercheurs trouvent de nouvelles solutions.
Recherches en cours

\section{sur la gestion des complexes parasitaires}

En résumé, les communications présentées indiquent que la recherche mise sur la chimie et sur les biotechnologies pour la protection de la culture du cotonnier, alors que les tentatives pour une approche plus systémique restent timides.

\section{Encore des travaux pour attester de l'efficacité du coton $\mathrm{Bt}$}

Alors que cela fait presque 15 ans que le coton Bt est cultivé à grande échelle, des travaux sont encore poursuivis pour statuer sur son efficacité. Les chenilles des capsules, ravageurs cibles des gènes $\mathrm{Bt}$, sont toujours présentes, parfois à un degré assez élevé dans les États du delta du Mississipi, ce qui est une différence notable avec ce qui est observé en Chine (Wu et al., 2008). Les orateurs indiquent qu'on ne doit pas les occulter, même si elles ne sont plus les ravageurs qui menacent le plus le coton. Deux communications ont ajouté que la résistance des chenilles des capsules au coton Bt n'a pas émergé.

En moyenne des dernières années, le gain de rentabilité du coton $\mathrm{Bt}$ tend à être négligeable par rapport au coton conventionnel protégé chimiquement. La forte augmentation du coût d'emploi de la technologie Bt (semences et royalties) en est une cause importante. Le retour au coton conventionnel pourrait paraître légitime, mais cela n'a pas été explicitement évoqué dans les communications.

\section{Persistance de la voie chimique pour contrôler les ravageurs cibles du coton $\mathrm{Bt}$}

Rappelons que c'est l'émergence de la résistance des chenilles des capsules aux insecticides à base de pyréthrinoïdes qui a été l'un des facteurs de la proposition du coton transgénique. Une communication a d'ailleurs porté sur le suivi de la résistance de ces ravageurs aux pyréthrinoïdes, alors que ces produits sont bien moins utilisés depuis le recours au coton Bt. Ce travail a cependant sa pertinence à un moment où le retour à la culture du coton conventionnel est évoqué.

Les limites actuelles du coton Bt semblent même ranimer la recherche de nouvelles familles de produits chimiques afin de contrôler ces ravageurs lépidoptères. Un nouveau produit commercial (famille Anthranilic Diamide) a été présenté pour son efficacité sur un large spectre de lépidoptères sur les grandes cultures (coton, maïs, soja...). L'homologation de ce produit serait déjà acquise pour la plupart des cultures, sauf pour le soja. Les résultats indiquent que deux applications de ce produit aboutissent à une protection équivalente à l'utilisation du coton Bt, mais l'information sur le coût reste évasive.

La proposition de produits chimiques à large spectre d'action marque un changement de stratégie des firmes de phytopharmacie par rapport à l'option de "frappe ciblée" sur des ravageurs précis. L'incidence possible de cette stratégie sur les changements des complexes des ravageurs n'est pas abordée.

\section{Contrôle chimique des ravageurs non-cibles du coton $\mathrm{Bt}$}

L'évaluation des pertes de récolte causées par les nouveaux ravageurs - principalement les piqueurs-suceurs et à un degré moindre les chenilles phylophages - et l'évaluation de l'efficacité des nouvelles molécules d'insecticide ont fait l'objet de 21 communications sur les 59 traitant du contrôle des ravageurs.

Cinq communications ont porté sur l'observation avérée de la perte de sensibilité des ravageurs piqueurs-suceurs (punaises, pucerons) vis-à-vis des insecticides utilisés depuis les années 2000 face à la recrudescence de leurs attaques. L'arrêt du recours à ces insecticides a donc été explicitement recommandé.

L'efficacité de nouveaux produits insecticides est actuellement évaluée par des expérimentations associant les chercheurs des firmes de phytopharmacie et les chercheurs des universités et les résultats sont présentés par les uns ou les autres. C'est un produit commercial de la famille des Sulfilimines qui semble avoir le vent en poupe, notamment visà-vis des deux espèces de punaises et des pucerons. L'efficacité contre les 
pucerons est telle qu'un orateur a même affirmé que le contrôle chimique des pucerons est ainsi résolu.

D’une manière générale, il faut souligner que les firmes ne cherchent pas à montrer que leurs produits sont meilleurs que ceux des concurrents. Elles insistent sur l'équivalence d'efficacité et sur la contribution à élargir la gamme des produits utilisables pour offrir flexibilité de choix et possibilité d'alternance dans l'utilisation des produits disponibles. Les travaux intègrent la mesure des effets sur un large spectre de ravageurs, avec cependant des doses différentes d'utilisation. Le large spectre est désormais présenté comme un avantage. De même, la variation des doses d'utilisation en fonction des ravageurs est avancée comme un atout de flexibilité et de compatibilité dans la mise en œuvre de programme de lutte intégrée.

\section{Contrôle des ravageurs par les biotechnologies}

Du côté des firmes de biotechnologie, la voie du coton transgénique est poursuivie avec la proposition de nouveaux types de variétés intégrant de nouveaux gènes.

C'est le cas de la firme Bayer avec le coton TwinLink, qui combine les deux gènes cry $1 \mathrm{Ab}$ et cry $2 \mathrm{Ae}$ contrôlant les ravageurs lépidoptères et le gène LibertyLink de cette firme pour la tolérance à un herbicide (à base de glufosinate d'ammonium). L'insertion d'un nouveau gène de tolérance au glyphosate de la même firme (gène GlyTol) est engagée pour donner lieu à de nouvelles variétés TwinLink/ GlyTol, qui auront la particularité de tolérer deux herbicides différents.

Syngenta, une autre multinationale en phytopharmacie et en biotechnologie, travaille de son côté à la combinaison du gène $\mathrm{Bt} c r y 1 A b$ et du gène Vip3A, homologué mais pas encore proposé à la commercialisation, pour la résistance aux ravageurs.

D'autres solutions envisagent une association plus complexe de trois gènes. C'est un chercheur de Monsanto qui annonce la commercialisation prochaine de Bollgard3, issue de la combinaison des deux gènes Bt utilisés dans Bollgard2 (cry1Ac et cry2Ab) et du gène Vip3A de Syngenta. Il était étonnant d'entendre un chercheur de Monsanto vanter les mérites du gène de Syngenta en termes de spectre plus large contre les ravageurs lépidoptères ! Monsanto est la seule firme à donner des résultats d'un nouveau gène $\mathrm{Bt}$ pour le contrôle d'une espèce de punaises bien que les toxines Bt ne paraissent pas adaptées a priori au mode d'ingestion des punaises. Les résultats sont prometteurs, mais il reste encore du chemin à parcourir avant la commercialisation.

\section{Exploration de pistes nouvelles pour le contrôle des nouveaux ravageurs}

Une première piste agronomique repose sur l'étude de la dynamique des populations des nouveaux ravageurs. L'effet négatif du voisinage du maïs sur l'infestation des punaises dans les champs de coton est mis en évidence, amenant la recommandation de réaliser des traitements contre les punaises sur le maïs en bordure des champs de coton.

Les premiers travaux sont engagés pour comprendre le déterminisme du système olfactif d'une espèce de punaise, qui serait déterminant pour la recherche de nourriture: sa compréhension peut ouvrir des voies de contrôle, en perturbant l'alimentation de ces ravageurs.

Une autre recherche repose sur l'exploitation de la vidéo haute définition pour filmer en continu les déplacements et l'alimentation d'une espèce de punaise, en fonction de l'âge et du sexe des insectes. L'idée est de comprendre qui, des mâles ou des femelles, et à quel stade, causent le plus de dégâts au cotonnier.

\section{Gestion des adventices résistantes au glyphosate}

Les chercheurs soulignent la nécessité de nouveaux produits chimiques, tout en considérant qu'il ne faut pas seulement miser sur les herbicides. Des techniques culturales, comme le retournement du sol, sont, dans certains cas, appropriées, même si cela pose un problème de compatibilité avec le zéro labour.

Les solutions chimiques proposées actuellement ne sont pas réellement efficaces. On a recouru, sans succès durable, aux herbicides résiduels ou de contact, en puisant dans les molécules existantes dont certaines déjà anciennes, comme le paraquat. À mesure que les phénomènes se développent et que de nouveaux produits sont proposés, de nouveaux programmes de lutte sont testés, qui associent plusieurs produits, en traitements de présemis et de post-émergence. Mais il est encore trop tôt pour se prononcer sur leur efficacité. Ces méthodes de lutte induisent bien sûr un coût supplémentaire.

Le nombre élevé d'espèces adventices résistant au glyphosate fait que leur contrôle doit combiner différentes techniques adaptées à la biologie de chaque adventice concernée. La connaissance de cette biologie devient un facteur primordial de l'efficacité des futurs moyens de lutte, d'autant plus que certaines plantes adventices, comme Ambrosia artemisiifolia, ont développé une sélection biologique avec une germination retardée, échappant ainsi à la période d'épandage des herbicides.

La mise en marché prochaine de nouvelles variétés transgéniques tolérantes à l'herbicide à base de $2-4 \mathrm{D}$ peut être une autre solution possible. L'enquête réalisée en temps réel ${ }^{3}$ lors d'un atelier avec les consultants indique une certaine méfiance à l'égard de ces nouvelles variétés, en raison des risques de dispersion de l'herbicide hors des champs traités.

\section{Conclusion}

Les conférences du Beltwide 2010 ont apporté une vision nouvelle des conséquences de l'utilisation des variétés de coton transgéniques aux États-Unis. Avec un recul de presque 15 années de culture, l'observation des changements dans les complexes d'ennemis de la culture du cotonnier et les solutions recherchées pour y faire face montrent que les effets positifs proclamés de l'utilisation de ces variétés ressemblent aujourd'hui à des illusions perdues dans trois domaines :

- les variétés de coton transgénique actuellement cultivées ne résolvent pas définitivement les problèmes des ennemis de la culture, parce que de nouveaux ennemis sont apparus (insectes ravageurs et plantes adventices). En conséquence, leur utilisation n'a pas permis de réduire durablement l'emploi des pesticides chimiques, redevenus nécessaires. Cet emploi est aujourd'hui coûteux et exige

\footnotetext{
3 Les organisateurs des conférences Beltwide, toujours à l'avant-garde des technologies, ont réalisé une enquête en temps réel en utilisant les produits de la firme elnstruction (http://www.einstruction.com/products/index. html).
} 
un haut degré de maîtrise technique, du fait de la nouveauté des molécules pesticides à utiliser et de la dépendance de leur efficacité aux conditions d'utilisation ; - le contrôle des ennemis de la culture par l'utilisation de variétés transgéniques est devenu globalement plus coûteux, tant par l'augmentation continue du prix des semences que par les pesticides à utiliser en complément. Dès lors, le sentiment de confort qui prévalait au début de cette utilisation est remplacé par un sentiment d'incertitude sur l'efficacité et la rentabilité de ce contrôle ; - les solutions de contrôle par la chimie ou les biotechnologies se révèlent être complémentaires alors qu'en Syrie, le recours aux produits chimiques a fortement diminué sans recourir aux variétés transgéniques (Alascar et Fok, 2009). De surcroit, la concurrence entre les firmes au sein de chacune des deux bran- ches est seulement virtuelle, soit par la non substituabilité des nouveaux pesticides chimiques, soit par l'entente possible entre les firmes de biotechnologies.

Les phénomènes observés aux États-Unis ne sont toutefois pas généralisables, parce qu'ils font référence à un cas extrême d'utilisation massive, simultanée et non coordonnée de variétés transgéniques de soja, de maïs et de coton, qui se succèdent sur les mêmes parcelles et dans les mêmes paysages. Il est donc risqué d'extrapoler ces phénomènes à d'autres régions du monde, même si de nouveaux ravageurs sont apparus en Chine et en Australie, tout comme des plantes adventices résistantes au glyphosate en Argentine et au Brésil. Face à ces phénomènes, un consultant a conclu à l'intérêt d'une approche systémique et coordonnée de l'utilisation des variétés transgéniques. Même si ce message n'a pas paru convaincre l'auditoire du Beltwide 2010, il nous paraît d'une importance primordiale pour bien appréhender la portée et les limites de l'utilisation des variétés transgéniques.

\section{Références}

Alascar H, Fok M. Politique cotonnière en Syrie: adaptation partielle et progressive à la mondialisation. Cah Agric 2009 ; 18 : 393-401.

Fernandez-Cornejo J, Caswell M. The First Decade of Genetically Engineered Crops in the United States. Washington (DC) : USDA Economic Research Service, 2006.

Hardee DD, Van Duyn JW, Layton MB, Bagwell RD. Bt Cotton Management of the Tobacco Budworm-Bollworm Complex. Washington (DC) : USDA Agricultural Research Service, 2001.

Wu K-M, Lu Y-H, Feng H-Q, Jiang Y-Y, Zhao J-Z. Suppression of Cotton Bollworm in Multiple Crops in China in Areas with Bt Toxin-Containing Cotton. Science 2008 ; 321 : 1676-8. 